\title{
Numerical atomic orbitals for linear-scaling calculations
}

\author{
Javier Junquera, ${ }^{1}$ Óscar Paz, ${ }^{1}$ Daniel Sánchez-Portal, ${ }^{2}$ and Emilio Artacho ${ }^{3}$ \\ ${ }^{1}$ Departamento de Física de la Materia Condensada, C-III, Universidad Autónoma, 28049 Madrid, Spain \\ ${ }^{2}$ Department of Physics and Materials Research Laboratory, University of Illinois, Urbana Illinois 61801, USA \\ ${ }^{3}$ Department of Earth Sciences, University of Cambridge, Downing Street, Cambridge CB2 3EQ, UK
}

(October 27, 2018)

\begin{abstract}
The performance of basis sets made of numerical atomic orbitals is explored in density-functional calculations of solids and molecules. With the aim of optimizing basis quality while maintaining strict localization of the orbitals, as needed for linear-scaling calculations, several schemes have been tried. The best performance is obtained for the basis sets generated according to a new scheme presented here, a flexibilization of previous proposals. The basis sets are tested versus converged plane-wave calculations on a significant variety of systems, including covalent, ionic and metallic. Satisfactory convergence (deviations significantly smaller than the accuracy of the underlying theory) is obtained for reasonably small basis sizes, with a clear improvement over previous schemes. The transferability of the obtained basis sets is tested in several cases and it is found to be satisfactory as well.
\end{abstract}

PACS Numbers: 71.15.Ap, 71.15.Mb

\section{INTRODUCTION}

In order to make intelligent use of the increasing power of computers for the first-principles simulation of ever larger and more complex systems, it is important to develop and tune linear-scaling methods, where the computational load scales only linearly with the number of atoms in the simulation cell. The present status of these methodsand their applications can be found in several reviews. sis sets made of localized wavefunctions represent a very sensible basis choice. It is not only the scaling what matters, however, the prefactor being also important for practical calculations. The prefactor depends significantly on two aspects of the basis: $(i)$ the number of basis functions per atom, and (ii) the size of the localization regions of these functions.

Atomic orbitals offer efficient basis sets since, even though their localization ranges are larger than those of some other methods, 3 the number of basis functions needed is usually quite small. The price to pay for this efficiency is the lack of systematics for convergence. Unlike with plane-wavet or real-space-gridl related methods, there is no unique way of increasing the size of the basis, and the rate of convergence depends on the way the basis is enlarged. This fact poses no fundamental difficulties, it just means that some effort is needed in the preparation of unbiased basis sets, in analogy to the extra work required to prepare pseudopotentials to describe the effect of core electrons.

Maximum efficiency is achieved by choosing atomic orbitals that allow convergence with small localization ranges and few orbitals. It is a challenge again comparable to the one faced by the pseudopotential community, where transferability and softness are sought 6 For atomic wavefunctions the optimization freedom is in the radial shape. Gaussian-type orbitals have been proposed for linear scaling, 9 spnnecting with the tradition of quantum chemistry. 1011 These bases are, however, quite rigid for the mentioned optimization, imposing either many gaussians or large localization ranges.

Numerical atomic orbitals (NAOs) are more flexible in this respect. Different ideas have been proposed in the literature, originally within tight-binding contexts concentrating on minimal (single- $\zeta$ ) bases. They are obtained by finding the eigenfunctions of the isolated atoms confined within spherical potential wells of different shapes, 1214 or directly modifying the eigenfunctions of the free atoms. 15 These schemes give strictly localized orbitals, i.e., orbitals that are strictly zero beyond given cutoff radii $r_{c}$. A first extension towards more complete basis sets was proposed using the excited states of the confined atoms, 16 but the quite delocalized character of many excited states made this approach inefficient unless very stringent confinement potentials were used.17

For multiple- $\zeta$, a better scheme was proposed hased on the split-valence idea of Quantum_Chemistry, 10101 but adapted to strictly localized NAOs. 18 In the same work, a systematic way was proposed to generate polarization orbitals suited for these basis sets. The scheme of Ref. 18 has proven to be quite efficient, systematic, and reasonable for a large variety of systems (for short reviews see Refs. 18, 19).

In this work we go beyond previous methodologies because of two main reasons: (i) It is always desirable to obtain the highest possible precision given the computational resources available, and (ii) it is important to know and show what is the precision attainable by NAO basis sets of reasonable sizes. None of these points was systematically addressed in this context before.

We explore these issues by variationally optimizing basis sets for a variety of condensed systems. The parameters defining the orbitals are allowed to vary freely to minimize the total energy of these systems. This energy 
is then compared with that of converged plane-wave calculations for exactly the same systems, including same density functional and pseudopotentials. The optimal basis sets are then tested monitoring structural and elastic properties of the systems.

The transferability of the basis sets optimized for particular systems is then checked by transfering them to other systems and testing the same energetical, structural and elastic parameters. Finally, the effect of localizing the orbitals tighter than what they variationally choose is explored on a demanding system.

\section{METHOD}

The calculations presented belpw were all done using depsity-functional theory 20.21 (DFT) in its localdensity 22 approximation (LDA). Core electrons were replaced by norm-conserying pseudopotentials $\theta$ in their fully separable form.23 The non-local partial-core exchange-correlation correction 24 was included for $\mathrm{Cu}$ to improve the description of the core-valence interactions.

Periodic boundary conditions were used for all systems. Molecules were treated in a supercell scheme allowing enough empty space between molecules to make intermolecular interactions negligible. For solid systems, integrations over the Brillouin zone were replaced by converged sums over selected $\vec{k}$-sets.25

Thus far the approximations are exactly the same for the two different sets of calculations performed in this work: based on NAOs and on plane-waves (PWs). The calculations using NAOs were ferformed with the SIESTA method, described elsewhere.1726 Besides the basis itself, the only additional approximation with respect to PWs is the replacement of some integrals in real space by sums in a finite 3D real-space grid, controlled hy one single parameter, the energy cutoff for the grid.26 This cutoff, which refers to the fineness of the grid, was converged for all systems studied here (200 Ry for all except for Si and $\mathrm{H}_{2}$, for which $80 \mathrm{Ry}$ and 100 Ry were used, respectively). Similarly, the PW calculations were done for converged PW cutoffs.27

Cohesive curves for the solids were obtained by fitting calculated energy values for differept unit-cell volumes to cubic, quartic and Murnaghan-like 28 curves, a procedure giving values to the lattice parameter, the bulk modulus and the cohesive energy of each system. The bulk moduli given by the Murnaghan and quartic fits deviate from each other by around 3\%, the Murnaghan values being the lowest and the ones shown in the tables. The deviations between Murnaghan and cubic fits are of the order of $7 \%$. The other cohesive parameters do not change appreciably with the fits.

The atomic-energy reference for the cohesive energy was taken from the atomic calculations within the same DFT and pseudopotentials, always converged in basis set. They are hence the same reference for NAOs and for PWs, the difference in cohesive energies between the two accounting for the difference in the total energy of the solid. The isolated-atom calculations included spin polarization.

\section{BASIS OF NUMERICAL ATOMIC ORBITALS}

The starting point of the atomic orbitals that conform the basis sets used here is the solution of Kohn-Sham's Hamiltonian for the isolated pseudo-atoms, solved in a radial grid, with the same approximations as for the solid or molecule (the same exchange-correlation functional and pseudopotential). A strict localization of the basis functions is ensured either by imposing a boundary condition, by adding a confining (divergent) potential, or by multiplying the free-atom orbital by a cutting function. We describe in the following three main features of a basis set of atomic orbitals: size, range, and radial shape.

\section{A. Size: number of orbitals per atom}

Following the nomenclature of Quantum Chemistry, we establish a hierarchy of basis sets, from single- $\zeta$ to multiple- $\zeta$ with polarization and diffuse orbitals, covering from quick calculations of low quality to high precision, as high as the finest obtained in Quantum Chemistry. A single- $\zeta$ (also called minimal) basis set (SZ in the following) has one single radial function per angular momentum channel, and only for those angular momenta with substantial electronic population in the valence of the free atom. It offers quick calculations and some insight on qualitative trends in the chemical bonding and other properties. It remains too rigid, however, for more quantitative calculations requiring both radial and angular flexibilization.

Starting by the radial flexibilization of SZ, a better basis is obtained by adding a second function per channel: double- $\zeta$ (DZ). Several schemes have been proposed to generate this second function. In Quantum Chemistry, the split valence 10,29 scheme is widely used: starting from the expansion in Gaussians of one atomic orbital, the most contracted gaussians are used to define the first orbital of the double- $\zeta$ and the most extended ones for the second. Another proposal defines the second $\zeta$ as the derivative of the first one with respect to occupation. 30 For strictly localized functions there was a first proposal 16 of using the excited states of the confined atoms, but it would work only for tight confinement. An extension of the split valence idea of Quantum Chemistry to strictly localized NAOs was proposed in Ref. 18 and has been used quite succesfully in a variety of systems. We follow this scheme in this work, which generalizes to multiple- $\zeta$ trivially. 
Angular flexibility is obtained by adding shells of higher angular momentum. Ways to generate these socalled polarization orbitals have been described in the literature, both for Gaussians 10 11 and for NAOs.18 In this work, however, they will be obtained variationally, as the rest, within the flexibilities described below.

\section{B. Range: cutoff radii of orbitals}

Strictly localized orbitals (zero beyond a cutoff radius) are used in order to obtain sparse Hamiltonian and overlap matrices for linear scaling. The traditional alternative to this is based on neglecting interactions when they fall below a tolerance or when the atoms are beyond some scope of neighbours. Its disadvantage is that it implies a deviation from the original Hilbert space, the total energy is no longer a variational magnitude, and produces numerical instabilities.

For the bases made of strictly localized orbitals, the problem is finding a balanced and systematic way of defining all the different radii, since both the precision and the computational efficiency in the calculations depend on them. A scheme was proposed 18 in which all radii were defined by one single parameter, the energy shift, i.e., the energy raise suffered by the orbital when confined. In this work, however, we step back from that systematic approach and allow the cutoff radii to vary freely in the optimization procedure (up to a maximum value of 8 a.u.).

\section{Shape}

Within the pseudopotential framework it is important to keep the consistency between the pseudopotential and the form of the pseudoatomic orbitals in the core region. This is done by using as basis orbitals the solutions of the same pseudopotential in the free atom. The shape of the orbitals at larger radii depends on the cutoff radius (see above) and op the way the localization is enforced. The first proposal 12 used an infinite square-well potential. It has been widely and succesfully used for minimal bases within the ab initio tight-binding scheme of Sankey and collaborators 12 using the FIREBALL program, but also for more flexible bases using the methodology of SIESTA.

This scheme has the disadavantage, however, of generating orbitals with a discontinuous derivative at $r_{c}$. This discontinuity is more pronounced for smaller $r_{c}$ 's and tends to disappear for long enough values of this cutoff. It does remain, however, appreciable for sensible values of $r_{c}$ for those orbitals that would be very wide in the free atom. It is surprising how small an effect such kink produces in the total energy of condensed systems (see below). It is, on the other hand, a problem for forces and stresses, especially if they are calculated using a (coarse) finite three-dimensional grid.
Another problem of this scheme is related to its defining the basis considering the free atoms. Free atoms can present extremely extended orbitals, their extension being, besides problematic, of no practical use for the calculation in condensed systems: the electrons far away from the atom can be described by the basis functions of other atoms.

Both problems can be addressed simultaneously by adding a soft confinement potential to the atomic Hamiltonian used to generate the basis orbitals: it smoothens the kink and contracts the orbital as variationally suited. Two soft confinement potentials have been proposed in the literature, both of the form $V(r)=V_{\mathrm{o}} r^{n}$, one for $n=2$ (Ref. 13) and the other for $n=6$ (Ref. 14). They present their own inconveniences, however. Firstly, there is no radius at which the orbitals become strictly zero, they have to be neglected at some point. Secondly, these confinement potentials affect the core region spoiling its adaptation to the pseudopotential.

This last problem affects a more traditional scheme as well, namely, the one based on the radial scaling of the orbitals by suitable scale factors. In addition to very basic bonding arguments, 31 it is soundly based on restoring virial's theorem for finite bases, in the case of coulombic potentials (all-electron calculations). 32 The pseudopotentials limit its applicability, allowing only for extremely small deviations from unity $(\sim 1 \%)$ in the scale factors obtained variationally (with the exception of hydrogen that can contract up to $25 \%$ ) 33

An alternative scheme has also been proposed:15 Instead of modifying the potential, it directly modifies the orbitals of the free atom. Following ideas of previous mixed-basis scheme 36 the atomic orbital is multiplied by $1-\exp \left[-\alpha\left(r-r_{c}\right)^{2}\right]$ for $r<r_{c}$ and zero otherwise. This scheme does provide strict localization beyond $r_{c}$, but introduces a different problem: for large $\alpha$ and small $r_{c}$ a bump appears in the orbital close to $r_{c}$, which becomes a discontinuity in the wave-function in the limit of zero $\alpha$.

In this work we propose a new soft confinement potential avoiding the mentioned deficiencies. It is flat (zero) in the core region, starts off at some internal radius $r_{i}$ with all derivatives continuous and diverges at $r_{c}$ ensuring the strict localization there. It is

$$
V(r)=V_{\mathrm{o}} \frac{e^{-\frac{r_{c}-r_{i}}{r-r_{i}}}}{r_{c}-r}
$$

In the following the different schemes are compared, their defining parameters being allowed to change variationally.

Finally, the shape of an orbital is also changed by the ionic character of the atom. Orbitals in cations tend to shrink, and they swell in anions. Introducing a $\delta Q$ in the basis-generating free-atom calculations gives orbitals better adapted to ionic situations in the condensed systems. 
TABLE I. Comparison of different confinement schemes on the cohesive properties of $\mathrm{MgO}$, for $\mathrm{SZ}$ and DZP basis sets. The generalization of the different schemes to DZP is done as explained in the text. Unconfined refers to using the unconfined pseudoatomic orbitals as basis. $a, B$, and $E_{c}$ stand for lattice parameter, bulk modulus, and cohesive energy, respectively. The PW calculations were performed with identical approximations as the NAO ones except for the basis. Augmented plane-wave (LAPW) results were taken from Ref. 44, and the experimental values from Ref. 43 .

\begin{tabular}{c|ccc|ccc}
\hline \hline Basis & \multicolumn{4}{|c|}{$\mathrm{SZ}$} & \multicolumn{4}{c}{$\mathrm{DZP}$} \\
scheme & $\begin{array}{c}a \\
(\AA)\end{array}$ & $\begin{array}{c}E_{c} \\
\mathrm{GPa})\end{array}$ & $\begin{array}{c}a \\
(\mathrm{eV})\end{array}$ & $\begin{array}{c}B \\
(\AA)\end{array}$ & $\begin{array}{c}E_{c} \\
(\mathrm{GPa})\end{array}$ & $(\mathrm{eV})$ \\
\hline Unconfined & 4.25 & 119 & 6.49 & & & \\
Sankey & 4.17 & 222 & 10.89 & 4.12 & 165 & 11.82 \\
Kenny & 4.16 & 228 & 11.12 & 4.12 & 163 & 11.84 \\
Porezag & 4.18 & 196 & 11.17 & 4.09 & 183 & 11.83 \\
Horsfield & 4.15 & 221 & 11.26 & 4.11 & 168 & 11.86 \\
This work & 4.15 & 226 & 11.32 & 4.10 & 167 & 11.87 \\
\hline PW & & & & 4.10 & 163 & 11.89 \\
LAPW & & & & 4.26 & 147 & 10.4 \\
Exp. & & & & 4.21 & 152 & 10.3 \\
\hline \hline
\end{tabular}

\section{OPTIMIZATION PROCEDURE}

Given a system and a basis size, the range and shape of the orbitals are defined as described above, depending on parameters. These are defined variationally: the energy is minimized with respect to all of them. As a robust and simple minimization method not requiring the evaluation of derivatives, we have chosen the downhill simplex method. 37

The parameters varied in an optimization are the following. Per atomic species there is a global $\delta Q$. Confinement is imposed differently for each angular momentum shell, with its corresponding parameters that depend on the scheme used. Hard confinement implies one parameter per shell $\left(r_{c}\right)$, and our soft confinement implies three $\left(r_{c}, r_{i}\right.$, and $\left.V_{\mathrm{o}}\right)$. One parameter $\left(V_{\mathrm{o}}\right)$ is needed only in the $r^{n}$-confinement schemes 13, 14 and two parameters in the scheme of Kenny et al 15 ( $r_{c}$ and the width of the cutting function). Finally, for each $\zeta$ beyond the first, there is a matching radius as defined in Ref. 18. The values obtained for the parameters in the optimizations described below can be obtained from the authors.

\section{RESULTS}

\section{A. Comparison of different confinement schemes}

Table If shows the performance for $\mathrm{MgO}$ of the different schemes described above for constructing localized atomic orbitals. The basis sets of both magnesium and oxygen were variationally optimized for all the schemes. $\mathrm{Mg}$ was chosen because the $3 s$ orbital is very extended in

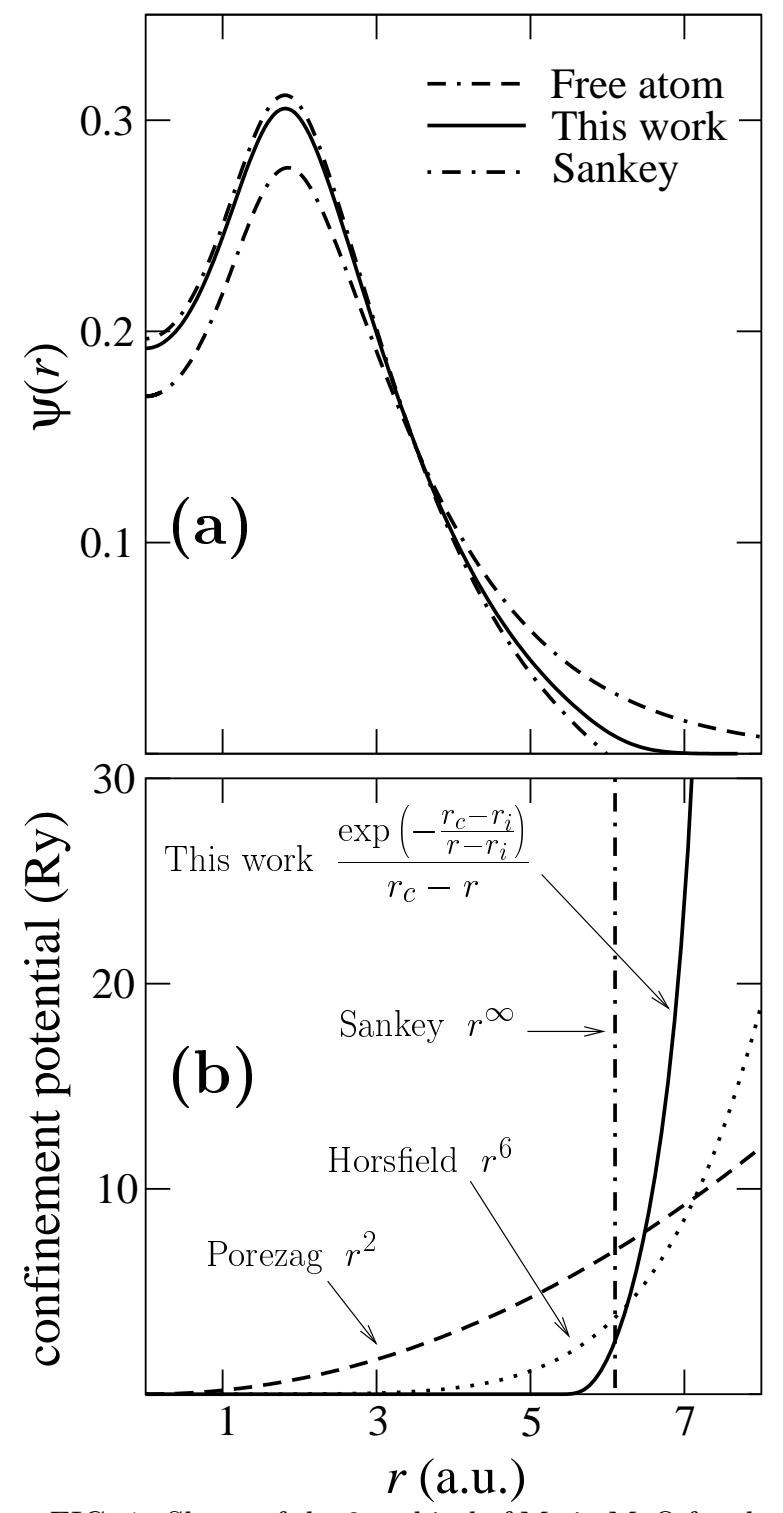

FIG. 1. Shape of the $3 s$ orbital of $\mathrm{Mg}$ in $\mathrm{MgO}$ for the different confinement schemes (a) and corresponding potentials (b).

the atom and both the kink and the confinement effects due to other orbitals are very pronounced. Results are shown for a SZ (single $s$ and $p$ channels for both species) and a DZP basis (double $s$ and $p$ channels plus a single $d$ channel). Fig. 1 shows the shape of the optimal $3 s$ orbital for the different schemes, and the shape of the confining potentials.

The following conclusions can be drawn from the results: $(i)$ Within the variational freedom offered here, the $3 s$ orbital of $\mathrm{Mg}$ wants to be confined to a radius of around 6.5 Bohr, irrespective of scheme, which is extremely short for the free atom. This confinement produces a pronounced kink in the hard scheme. (ii) The total energy is relatively insensitive to the scheme used to generate the basis orbitals, as long as there is effective confinement. (iii) The basis made of unconfined atomic 
TABLE II. Basis comparisons for bulk Si. $a, B$, and $E_{c}$ stand for lattice parameter (in $\AA$ ), bulk modulus (in GPa), and cohesive energy (in eV), respectively. SZ, DZ, and TZ stand for single- $\zeta$, double- $\zeta$, and triple- $\zeta$. P stands for polarized, DP for doubly polarized. LAPW results were taken from Ref. 48, and the experimental values from Ref. 39 .

\begin{tabular}{ccccccccccc}
\hline \hline & SZ & DZ & TZ & SZP & DZP & TZP & TZDP & PW & LAPW & Exp \\
\hline$a$ & 5.52 & 5.49 & 5.48 & 5.43 & 5.40 & 5.39 & 5.39 & 5.38 & 5.41 & 5.43 \\
$B$ & 85 & 87 & 85 & 97 & 97 & 97 & 97 & 96 & 96 & 98.8 \\
$E_{c}$ & 4.70 & 4.83 & 4.85 & 5.21 & 5.31 & 5.32 & 5.34 & 5.40 & 5.28 & 4.63 \\
\hline \hline
\end{tabular}

orbitals is substantially worse than any of the others. (iv) The pronounced kink obtained in Sankey's hard confinement scheme is not substantially affecting the total energy as compared with the other schemes. It does perturb, however, by introducing inconvenient noise in the energy variation with volume and other external parameters, and especially in the derivatives of the energy. $(v)$ The scheme proposed in this work is variationally slightly better than the other ones, but not significantly. Its main advantage is the avoidance of known problems. In the remainder of the paper, the confinement proposed in this work will be used unless otherwise specified.

\section{B. Basis convergence}

Table II shows how NAO bases converge for bulk silicon. This is done by comparing different basis sizes, each of them optimized. The results are compared to converged (50 Ry) PW results (converged basis limit) keeping the rest of the calculation identical. They are also compared to all-electron LDA results 48 to compare basis errors with the ones produced by the pseudopotentials. Experiment gives a reference to the accuracy of the underlying LDA. Fig. 2(a) shows the cohesion curves for this system.

The comparisons above are made with respect to the converged-basis limit, for which we used PWs up to very high cutoffs. It is important to distinguish this limit from the PW calculations at lower cutoffs, as used in many computations. To illustrate this point, Fig. 2(b) compares the energy convergence for PWs and for NAOs. Even though the convergence of NAO results is a priori not systematic with the way the basis is enlarged, the sequence of bases presented in the Figure shows a nice convergence of total energy with respect to basis size (the number of basis functions per atom are shown in parenthesis in the Figure): the convergence rate is similar to the one of PWs (DZP has three times more orbitals than $\mathrm{SZ}$, and a similar factor is found for their equivalents in PWs). For the particular case of Si, Fig. 2 shows that the polarization orbitals ( $3 d$ shell) are very important for convergence, more than the doubling of the basis. This fact is observed from the stabilization of SZP with respect to SZ, which is much larger than for DZ.
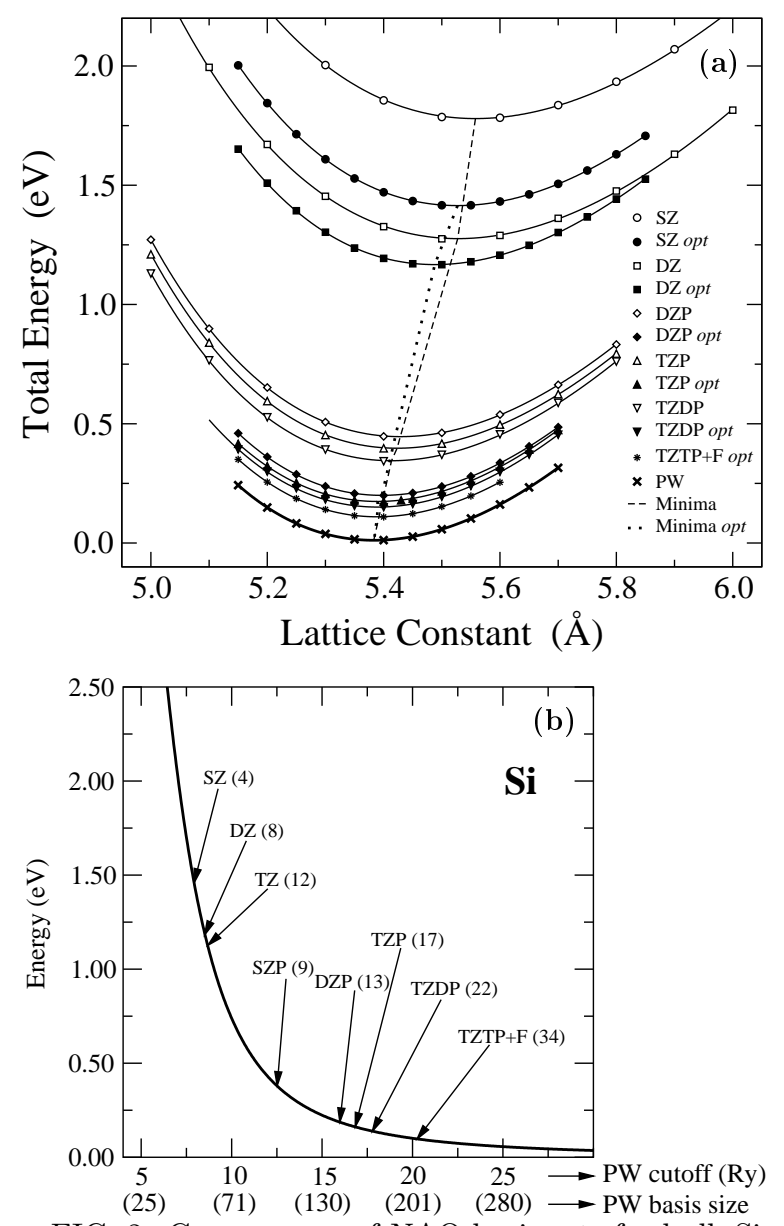

FIG. 2. Convergence of NAO basis sets for bulk Si.

(a) Cohesive curve for different basis sets. The lowest curve shows the PW results, filled symbols the NAO bases of this work (opt), and open symbols the NAO bases following Ref. 18. Basis labels are like in Table II, F standing for an extra $f$-shell.

(b) Comparison of NAO convergence with PW convergence. In parenthesis the number of basis functions per atom. 
TABLE III. Equivalent PW cutoff ( $\left.E_{\text {cut }}\right)$ to optimal DZP bases for different systems. Comparison of number of basis functions per atom for both bases. For the molecules, a cubic unit cell of $10 \AA$ of side was used.

\begin{tabular}{cccc}
\hline \hline System & \# funct. DZP & \# funct. PW & $E_{\text {cut }}(\mathrm{Ry})$ \\
\hline $\mathrm{H}_{2}$ & 5 & 11296 & 34 \\
$\mathrm{O}_{2}$ & 13 & 45442 & 86 \\
$\mathrm{Si}$ & 13 & 144 & 16 \\
Diamond & 13 & 284 & 59 \\
$\alpha$-quartz & 13 & 923 & 76 \\
\hline \hline
\end{tabular}

Fig. 22 shows that an atomic basis requires ten times less functions than its (energetically) equivalent PW basis, being $\mathrm{Si}$ the easiest system for PWs. For other systems the ratio is much larger, as shown in Table III.

It is important to stress that deviations smaller than the ones due to the pseudopotential or the DFT used are obtained with a relatively modest basis size as DZP. This fact is clear in Table II for $\mathrm{Si}$, and in Table IV for other systems. Table IV summarizes the cohesion results for a variety of solids of different chemical kind. They are obtained with optimal DZP basis sets. It can be observed that DZP offers results in good agreement with converged-basis numbers, showing the convergence of properties other than the total energy. The deviations are similar or smaller than those introduced by LDA or by the pseudopotential 46

\section{TRANSFERABILITY}

To what extent do optimal bases keep their performance when transfered to different systems than the ones they were optimized for? This is an important question, since if the performance does not suffer significantly, one can hope to tabulate basis sets per species, to be used for whatever system. If the transferability is not satisfactory, a new basis set should then be obtained variationally for each system to be studied. Of course the transferability increases with basis size, since the basis has more flexibility to adapt to different environments. A thorough study of transferability, therefore, implies many calculations in different systems, for different bases and different basis sizes. In this work we limit ourselves to try it on DZP bases for a few representative systems.

Satisfactory transferability has been obtained when checking in $\mathrm{MgO}$ the basis set optimized for $\mathrm{Mg}$ bulk and $\mathrm{O}$ in a water molecule. Similarly the basis for O has been tested in $\mathrm{H}_{2} \mathrm{O}$ and $\mathrm{O}_{2}$, and the basis for $\mathrm{C}$ in graphite and diamond. Again, the results show deviations due to the basis that are smaller than the errors introduced by the pseudopotentials and/or the DFT functional. The results are shown in Table $\mathrm{V}$.

Table VI shows the results for the structural parameters of $\mathrm{SiO}_{2}$ in its $\alpha$-quartz structure. The DZP numbers have been obtained for a basis that was optimized not
TABLE IV. Basis comparisons for different solids. $a, B$, and $E_{c}$ stand for lattice parameter (in $\AA$ ), bulk modulus (in $\mathrm{GPa}$ ), and cohesive energy (in eV), respectively.

\begin{tabular}{|c|c|c|c|c|c|c|}
\hline & & Exp & LAPW & Other PW & PW & DZP \\
\hline \multirow[t]{3}{*}{$\overline{\mathrm{Au}}$} & $a$ & $4.08^{\mathrm{a}}$ & $4.05^{\mathrm{b}}$ & $4.07^{\mathrm{i}}$ & 4.05 & 4.07 \\
\hline & $B$ & $173^{\mathrm{a}}$ & $198^{\mathrm{b}}$ & $190^{\mathrm{i}}$ & 195 & 188 \\
\hline & $E_{c}$ & $3.81^{\mathrm{a}}$ & - & - & 4.36 & 4.13 \\
\hline \multirow[t]{3}{*}{$\overline{\mathrm{MgO}}$} & $a$ & $4.21^{\mathrm{C}}$ & $4.26^{\mathrm{d}}$ & - & 4.10 & 4.11 \\
\hline & $B$ & $152^{\mathrm{c}}$ & $147^{\mathrm{d}}$ & - & 164 & 167 \\
\hline & $E_{c}$ & $10.30^{\mathrm{c}}$ & $10.40^{\mathrm{d}}$ & - & 11.89 & 11.87 \\
\hline \multirow[t]{3}{*}{$\overline{\mathrm{C}}$} & $a$ & $3.57^{\mathrm{a}}$ & $3.54^{\mathrm{f}}$ & $3.54^{\mathrm{e}}$ & 3.53 & 3.54 \\
\hline & $B$ & $442^{\mathrm{a}}$ & $470^{\mathrm{f}}$ & $436^{\mathrm{e}}$ & 459 & 453 \\
\hline & $E_{c}$ & $7.37^{\mathrm{a}}$ & $10.13^{\mathrm{f}}$ & $8.96^{\mathrm{e}}$ & 8.89 & 8.81 \\
\hline \multirow[t]{3}{*}{$\overline{\mathrm{Si}}$} & $a$ & $5.43^{\mathrm{a}}$ & $5.41^{\mathrm{g}}$ & $5.38^{\mathrm{e}}$ & 5.38 & 5.40 \\
\hline & $B$ & $99^{\mathrm{a}}$ & $96^{\mathrm{g}}$ & $94^{\mathrm{e}}$ & 96 & 97 \\
\hline & $E_{c}$ & $4.63^{\mathrm{a}}$ & $5.28^{\mathrm{g}}$ & $5.34^{\mathrm{e}}$ & 5.40 & 5.31 \\
\hline \multirow[t]{3}{*}{$\overline{\mathrm{Na}}$} & $\bar{a}$ & $4.23^{\mathrm{a}}$ & $4.05^{\mathrm{k}}$ & $3.98^{\mathrm{e}}$ & 3.95 & 3.98 \\
\hline & $B$ & $6.9^{\mathrm{a}}$ & $9.2^{\mathrm{k}}$ & $8.7^{\mathrm{e}}$ & 8.8 & 9.2 \\
\hline & $E_{c}$ & $1.11^{\mathrm{a}}$ & $1.44^{\mathrm{h}}$ & $1.28^{\mathrm{e}}$ & 1.22 & 1.22 \\
\hline \multirow[t]{3}{*}{$\overline{\mathrm{Cu}}$} & $a$ & $3.60^{\mathrm{a}}$ & $3.52^{\mathrm{b}}$ & $3.56^{\mathrm{e}}$ & - & 3.57 \\
\hline & $B$ & $138^{\mathrm{a}}$ & $192^{\mathrm{b}}$ & $172^{\mathrm{e}}$ & - & 165 \\
\hline & $E_{c}$ & $3.50^{\mathrm{a}}$ & $4.29^{\mathrm{j}}$ & $4.24^{\mathrm{e}}$ & - & 4.37 \\
\hline \multirow[t]{3}{*}{$\overline{\mathrm{Pb}}$} & $a$ & $4.95^{\mathrm{a}}$ & - & 4.88 & - & 4.88 \\
\hline & $B$ & $43^{\mathrm{a}}$ & - & 54 & - & 64 \\
\hline & $E_{c}$ & $2.04^{\mathrm{a}}$ & - & 3.77 & - & 3.51 \\
\hline
\end{tabular}

${ }^{\mathrm{a}}$ C. Kittel, Ref. 39

${ }^{\mathrm{b}}$ A. Khein, D. J. Singh, C.J. Umrigar, Ref. 40

${ }^{\mathrm{c}}$ F. Finocchi, J. Goniakowski, C. Noguera, Ref. 43

${ }^{\mathrm{d}}$ J. Goniakowski, C. Noguera, Ref. 44

${ }^{\mathrm{e}}$ M. Fuchs, M. Bockstedte, E. Pehlke, M. Scheffler, Ref. 46

${ }^{\mathrm{f}}$ N. A. W. Holzwarth et al, Ref. 47

${ }^{\mathrm{g}}$ C. Filippi, D. J. Singh, and C. J. Umrigar, Ref. 48

${ }^{\mathrm{h}}$ M. Sigalas et al., Ref. 49

${ }^{\mathrm{i}}$ B. D. Yu and M. Scheffler, Ref. 41

${ }^{\mathrm{j}}$ P. H. T. Philipsen and E. J. Baerends, Ref. 42

${ }^{\mathrm{k}}$ J. P. Perdew et al., Ref. 45 
TABLE V. Transferability of basis sets. "Transf" stands for the DZP basis transfered from other systems, while "Opt" refers to the DZP basis optimized for the particular system. For $\mathrm{MgO}$ the basis was transfered from bulk $\mathrm{Mg}$ and an $\mathrm{H}_{2} \mathrm{O}$ molecule, for graphite the basis was transfered from diamond, and for $\mathrm{H}_{2} \mathrm{O}$ it was taken from $\mathrm{H}_{2}$ and $\mathrm{O}_{2}$. $a, B$, and $E_{c}$ stand for lattice parameter, bulk modulus, and cohesive energy, respectively. $\Delta E$ stands for the energy difference per atom between graphite and a graphene plane. $E_{b}$ is the binding energy of the molecule.

\begin{tabular}{|c|c|c|c|c|}
\hline System & Basis & \multicolumn{3}{|c|}{ Properties } \\
\hline $\mathrm{MgO}$ & & $a(\AA)$ & $B(\mathrm{GPa})$ & $E_{c}(\mathrm{eV})$ \\
\hline & Transf & 4.13 & 157 & 11.81 \\
\hline & Opt & 4.10 & 167 & 11.87 \\
\hline & PW & 4.10 & 164 & 11.89 \\
\hline & LAPW & 4.26 & 147 & 10.40 \\
\hline & Exp & 4.21 & 152 & 10.30 \\
\hline Graphite & & $a(\check{A})$ & $c(\check{A})$ & $\Delta E(\mathrm{meV})$ \\
\hline & Transf & 2.456 & 6.50 & 38 \\
\hline & $\mathrm{PW}^{\mathrm{a}}$ & 2.457 & 6.72 & 24 \\
\hline & $\operatorname{Exp}^{b}$ & 2.456 & 6.674 & $23^{\mathrm{c}}$ \\
\hline $\mathrm{H}_{2} \mathrm{O}$ & & $d_{\mathrm{O}-\mathrm{H}}(\check{\mathrm{A}})$ & $\theta_{\mathrm{O}-\mathrm{H}-\mathrm{O}}(\mathrm{deg})$ & $\overline{E_{b}(\mathrm{eV})}$ \\
\hline & Transf & 0.975 & 105.0 & 12.73 \\
\hline & Opt & 0.972 & 104.5 & 12.94 \\
\hline & PW & 0.967 & 105.1 & 13.10 \\
\hline & $\mathrm{LAPW}^{\mathrm{d}}$ & 0.968 & 103.9 & 11.05 \\
\hline & $\operatorname{Exp}^{\mathrm{e}}$ & 0.958 & 104.5 & 10.08 \\
\hline
\end{tabular}

${ }^{\mathrm{a}}$ M. C. Schabel, Bef.55

${ }^{\mathrm{b}}$ Y. Baskin, Ref 56

${ }^{\mathrm{c}}$ L. A. Girifalco Ref 57

${ }^{\mathrm{d}} \mathrm{P}$. Serena, Ref 58

${ }^{\mathrm{e}} \mathrm{G}$. Herzberg, Ref. $\mathrm{g}$

TABLE VI. Performance of the basis of Si and O as optimized in c-Si and in a water molecule, respectively, for the structural parameters of $\alpha$-quartz.

\begin{tabular}{lcccccc}
\hline \hline & $\operatorname{Exp}^{\mathrm{a}}$ & $\mathrm{PW}^{\mathrm{b}}$ & $\mathrm{PW}^{\mathrm{c}}$ & $\mathrm{PW}^{\mathrm{d}}$ & $\mathrm{PW}^{\mathrm{e}}$ & $\mathrm{DZP}$ \\
\hline$a(\AA)$ & 4.92 & 4.84 & 4.89 & 4.81 & 4.88 & 4.85 \\
$c(\AA)$ & 5.41 & 5.41 & 5.38 & 5.32 & 5.40 & 5.38 \\
$d_{\mathrm{Si}-\mathrm{O}}^{1}(\AA)$ & 1.605 & 1.611 & 1.60 & 1.605 & - & 1.611 \\
$d_{\mathrm{Si}-\mathrm{O}}^{2}(\AA)$ & 1.614 & 1.617 & 1.60 & 1.605 & - & 1.611 \\
$\alpha_{\mathrm{Si}-\mathrm{O}-\mathrm{Si}}(\mathrm{deg})$ & 143.7 & 140.2 & - & 139.0 & - & 140.0 \\
\hline \hline
\end{tabular}

${ }^{a}$ L. Levien, C. T. Prewitt and D. J. Weidner, Ref. 54.

${ }^{\mathrm{b}} \mathrm{P}$. Sautet (unpublished) using ultrasoft pseudopotentials.61

${ }^{\mathrm{c}}$ D. R. Hamann, Ref. 52

${ }^{\mathrm{d}}$ G.-M.Rignanese et al., Ref. 53 .

${ }^{\mathrm{e}} \mathrm{F}$. Liu et al., Ref. 50, using ultrasoft pseudopotentials.
TABLE VII. Tightening the confinement of the basis in $\alpha$-quartz.

\begin{tabular}{ccc|ccc|ccccc}
\hline \hline \multicolumn{2}{c|}{$r_{c}^{\mathrm{Si}}$ (a.u.) } & \multicolumn{2}{|c|}{$r_{c}^{\mathrm{O}}$ (a.u.) } & $a$ & $c$ & \multicolumn{2}{c}{$d_{\mathrm{Si}-\mathrm{O}}^{1}$} & $d_{\mathrm{Si}-\mathrm{O}}^{2}$ & $\alpha_{\mathrm{Si}-\mathrm{O}-\mathrm{Si}}$ \\
$s$ & $p$ & $d$ & $s$ & $p$ & $d$ & $(\AA)$ & $(\AA)$ & $(\AA)$ & $(\AA)$ & $(\mathrm{deg})$ \\
\hline 8.0 & 8.0 & 8.0 & 8.0 & 8.0 & 8.0 & 4.85 & 5.38 & 1.611 & 1.612 & 140.0 \\
6.0 & 6.0 & 6.0 & 8.0 & 8.0 & 8.0 & 4.85 & 5.35 & 1.607 & 1.608 & 140.0 \\
6.0 & 6.0 & 6.0 & 5.0 & 5.0 & 5.0 & 4.74 & 5.29 & 1.610 & 1.610 & 134.0 \\
6.0 & 6.0 & 6.0 & 4.5 & 4.5 & 4.5 & 4.69 & 5.26 & 1.610 & 1.610 & 132.0 \\
6.0 & 6.0 & 6.0 & 5.0 & 6.5 & 4.0 & 4.84 & 5.36 & 1.607 & 1.608 & 139.7 \\
5.6 & 6.3 & 4.2 & 4.0 & 5.3 & 2.8 & 4.81 & 5.34 & 1.607 & 1.610 & 138.2 \\
\hline \hline
\end{tabular}

for $\alpha$-quartz itself, but for bulk silicon for the Si basis and for the water molecule for the $\mathrm{O}$ basis. $\mathrm{SiO}_{2}$ was chosen because its being quite sensitive to many approximations and in particular to the basis set. It was hard to converge for previous NAO schemes, 18 giving 60 typically longer Si-O bonds (with deviations of around 1.5\%) and smaller unit cells (deviations of around $1.5 \%$ and $2 \%$ for the $a$ and $c$ parameters, respectively). The results of Table VI are very satisfactory, showing $(i)$ the good performance of NAOs, $(i i)$ their transferability in this case, and (iii) the improvement of the basis sets proposed here over previous bases.

\section{LIMITING THE RANGE}

In this work we have concentrated on variationally optimized basis sets, allowing the cutoff radii for the different orbitals to vary freely, as long as the orbitals remained strictly localized. This was done in the spirit of exploring the capabilities of the NAO basis sets. Some orbitals demanded reasonably short values of $r_{c}$, others chose long ranges. As mentioned earlier, the range of the orbitals is important for the efficiency in the calculations. Therefore, further work will be very important to explore the possibility of enforcing smaller ranges in reasonably balanced ways and its effect on the precision. A systematic study in this direction will be subject of future work, we have limited ourselves here to illustrate the nature of the problem in the particular example of $\alpha$-quartz.

The basis has been optimized as before ( $\mathrm{Si}$ in bulk $\mathrm{Si}$ and $\mathrm{O}$ in $\mathrm{H}_{2} \mathrm{O}$ ), but imposing now tighter $r_{c}$ 's. The results are summarized in Table VII. The constraining of Si orbitals to 6.0 a.u. affects the geometry only slightly, whilst the contraction of the $\mathrm{O}$ orbitals to 5.0 a.u. implies a substantial contraction of the cell due to the decrease of Si-O-Si angle rather than the shortening of the $\mathrm{Si}-\mathrm{O}$ bond. Note that, from an atomic perspective, the confinement of $\mathrm{Si}$ to 6 a.u. is tighter than the 5 a.u. confinement of $\mathrm{O}$.

Allowing for different $r_{c}$ 's for the different channels we observe that the shrinking of the cell is avoided keeping a long $p$ orbital for $\mathrm{O}$, the $s$ and $d$ remaining comparably shorter, indicating the (expected 18 ) different "compressibilities" of the different orbitals. As a candidate of unify- 
ing criterion, we tested the simple definition of $r_{c}$ for each channel as the value of the radius at which the unconstrained orbital would have a defined value $\left(0.01\right.$ a.u. $\left.{ }^{-3}\right)$. Even though the energy raise was appreciable (about 70 meV per atom), the geometry retained an acceptable precision, the orbitals being quite short, thus allowing quite efficient calculations. Further work is, however, needed to explore in detail this and other possibilities.

\section{CONCLUSIONS}

The variational optimization of NAO basis sets for different systems allows us to draw the following conclusions. ( $i$ ) The performance of NAO basis sets of modest size as DZP is very satisfactory for the systems tried. For this basis size, the deviation from basis convergence is smaller than errors due to the pseudopotential or DFT used. (ii) The bases obtained here represent a substantial improvement over previous NAO basis sets. In particular, the optimization in condensed systems offers better and more efficient bases than purely atomic schemes. (iii) The radial shapes of the orbitals obtained as proposed in this work offer better bases than previous schemes from a variational point of view, albeit not a substantial difference is obtained. (iv) The elimination of the discontinuity in the derivative, while retaining strict localization and leaving the core region untouched, gives bases of better quality from the point of view of the energy, its derivatives, and computational efficiency. $(v)$ The bases obtained showed enough transferability to expect that a basis tabulation would be useful, and that the optimization of the basis for each particular system will not be necessary. Finally, the selective sensitivity to orbital-range tightening has been shown, making clear the need of further work systematically to control the cutoff radii for improving efficiency without loss of precision.

\section{ACKNOWLEDGMENTS}

We acknowledge discussions and input from J. M. Soler, E. Anglada, Z. Barandiarán, L. Seijo, P. Ordejón, and A. García. This work was supported by the Spanish Dirección General de Investigación under grant BFM2000-1312 and by the Fundación Ramón Areces of Spain.

\footnotetext{
${ }^{1}$ P. Ordejón, Comp. Mat. Sci. 12, 157 (1998).

${ }^{2}$ S. Goedecker, Rev. Mod. Phys. 71, 1085 (1999).
}

${ }^{3}$ E. Hernández and M. J. Gillan, Phys. Rev. B 51, 10157 (1995); E. Hernández, M. J. Gillan and C. M. Goringe, Phys. Rev. B 53, 7147 (1996).

${ }^{4}$ M. C. Payne, M. P. Teter, D. C. Allan, T. A. Arias and J. D. Joannopoulos, Rev. Mod. Phys. 64, 1045 (1992).

${ }^{5}$ J. R. Chelikowski, N. Troullier, and Y. Saad, Phys. Rev. Lett., 72, 1240, (1994); E. L. Briggs, D. J. Sullivan, and J. Bernholc, Phys. Rev. B, 52, R5471, (1995).

${ }^{6}$ N. Troullier and J. L. Martins, Phys. Rev. B. 43, 1993 (1991).

${ }^{7}$ C. White, B. Johnson, P. Gill and M. Head-Gordon, Chem. Phys. Lett. 230, 8 (1994).

${ }^{8}$ Konstantin N. Kudin and Gustavo Scuseria Phys. Rev. B. 61, 16440 (2000).

${ }^{9}$ G. Lippert, J. Hutter, and M. Parrinello, Mol. Phys. 92, 477 (1997).

10 S. Huzinaga, Computer Physics Reports, 2, 279 (1985); S. Huzinaga et al., Gaussian Basis Sets for Molecular Calculations, (Elsevier Sci. Publ. Co., Amsterdam, 1984).

${ }^{11}$ R. Poirier, R. Kari, and R. Csizmadia, Handbook of Gaussian Basis Sets, (Elsevier Sci. Publ. Co., Amsterdam, 1985) and references therein.

12 O. F. Sankey and D. J. Niklewski, Phys. Rev. B. 40, 3979 (1989).

13 D. Porezag, Th. Frauenheim, Th. Köhler, G. Seifert and R. Kaschner, Phys. Rev. B. 51, 12947 (1995).

${ }^{14}$ Andrew P. Horsfield, Phys. Rev. B. 56, 6594 (1997).

15 S. D. Kenny, A. P. Horsfield, Hideaki Fujitani, Phys. Rev. B 62, 4899 (2000).

${ }^{16}$ D. Sánchez-Portal, E. Artacho and J. M. Soler, J. Phys: Condensed Matter 8, 3859 (1996).

17 D. Sánchez-Portal, E. Artacho, P. Ordejón, and J. M. Soler, Int. J. Quantum Chem. 65, 453 (1997).

${ }^{18}$ E. Artacho, D. Sánchez-Portal, P. Ordejón, A. García and J. M. Soler, Phys. Stat. Sol. (b), 215, 809 (1999).

19 P. Ordejón, Phys. Stat. Sol. (b) 217, 335 (2000).

${ }^{20}$ P. Hohenberg and W. Kohn, Phys. Rev., 136, 864 (1964).

${ }^{21}$ W. Kohn and L. J. Sham, Phys. Rev. 140, A1133 (1965).

22 J. P. Perdew and A. Zunger, Phys. Rev. B, 23, 5048 (1981).

${ }^{23}$ L. Kleinman and D. M. Bylander, Phys. Rev. Lett., 48, 1425 (1982).

${ }^{24}$ S. G. Louie, S. Froyen, and M. L. Cohen, Phys. Rev. B 26, 1738 (1992).

${ }^{25}$ H. J. Monkhorst, and D. J. Pack, Phys. Rev. B 13, 5188 (1976).

${ }^{26}$ P. Ordejón, E. Artacho, and J. M. Soler, Phys. Rev. B 53, R10441 (1996).

27 A plane wave cutoff of $100 \mathrm{Ry}$ was used for $\mathrm{MgO}$ and diamond; $62 \mathrm{Ry}$ for $\mathrm{Au}$; $50 \mathrm{Ry}$ for $\mathrm{Si}$ and $\mathrm{Na}$; $90 \mathrm{Ry}$ for $\alpha$-quartz and $140 \mathrm{Ry}$ for $\mathrm{H}_{2} \mathrm{O}$.

${ }^{28}$ F. D. Murnaghan, Proc. Natl. Acad. Sci., 30, 244 (1944).

${ }^{29}$ A. Szabo and N. Ostlund Modern Quantum Chemistry, (MacMillan, New York, 1982).

${ }^{30}$ G. Lippert, J. Hutter, P. Ballone, and M. Parrinello, J. Phys. Chem. 100, 6231 (1997).

${ }^{31}$ C. Cohen-Tannoudji, B. Diu, F. Laloë Quantum Mechanics (Hermann and John Wiley and sons, Paris, 1973).

32 I. N. Levine, Quantum Chemistry, 3rd Ed. (Allyn and Bacon, Boston, 1983).

${ }^{33}$ In the context of NAOs it has been used for SZ bases. In 
liquid silicon simulations 34 variational contractions of $2 \%$ for the $3 p$ and $0 \%$ the $3 s$ orbitals of $\mathrm{Si}$ were used. For carbon in fullerene 35 the contraction was of $5 \%$ for $2 s$ and $2 p$. And the $1 s$ orbital of hydrogen contracted $3520 \%$ in $\mathrm{H}_{2}$ and $25 \%$ in $\mathrm{H}_{2} \mathrm{O}$.

${ }^{34}$ G. Fabricius, E. Artacho, D. Sánchez-Portal, P. Ordejón, D. A. Drabold, and J. M. Soler, Phys. Rev. B 60, 16283 (1999).

${ }^{35}$ E. Artacho, unpublished.

${ }^{36}$ C. Elsaesser, N. Takeuchi, K. M. Ho, C. T. Chan, P. Braun, and M. Fähnle, J. Phys.: Condens. Matter 2, 4371 (1990).

${ }^{37}$ W. H. Press, B. P. Flannery, S. A. Teukolsky, W. T. Vetterling, Numerical Recipes: the art of scientific computing (Cambridge University Press, Cambridge, 1986).

${ }^{38}$ Values for the parameters can be obtained upon request.

${ }^{39}$ C. Kittel, Introduction to Solid State Physics (John Wiley \& Sons, New York, 1986).

${ }^{40}$ Alexander Khein, D. J. Singh and C. J. Umrigar, Phys. Rev. B, 51, 4105 (1995).

${ }^{41}$ B. D. Yu and M. Scheffler, Phys. Rev. B, 56, R15569 (1997).

42 P. H. T. Philipsen and E. J. Baerends, Phys. Rev. B, 54, 5326 (1996).

43 Fabio Finocchi, Jacek Goniakowski and Claudine Noguera, Phys. Rev. B, 59, 5178 (1999).

44 Jacek Goniakowski and Claudine Noguera, Phys. Rev. B, 60, 16120 (1999).

${ }^{45}$ J. P. Perdew, J. A. Chevary, S. H. Vosko, K. A. Jackson, M. R. Pederson, D. J. Singh and C. Fiolhais, Phys. Rev. B, 46, 6671 (1992).

${ }^{46}$ M. Fuchs, M. Bockstedte, E. Pehlke, and M. Scheffler Phys. Rev. B, 57, 2134 (1998).

${ }^{47}$ N. E. W. Holzwarth, G. E. Matthews, R. B. Dunning, A. R. Tackett, and Y. Zeng, Phys. Rev. B, 55, 2005 (1997).

${ }^{48}$ C. Filippi, D. J. Singh and C. J. Umrigar, Phys. Rev. B, 50, 14947 (1994).

${ }^{49}$ M. Sigalas, N. C. Bacalis, D. A. Papaconstantopoulos, M. J. Mehl, and A. C. Switendick, Phys. Rev. B, 42, 11637 (1990).

${ }^{50}$ Feng Liu, Stephen H. Garofalini, Dominic King-Smith, and David Vanderbilt Phys. Rev. B, 49, 12528 (1994).

${ }^{51}$ N. R. Keskar and J. R. Chelikowsky, Phys. Rev. B, 46, 1 (1992).

${ }^{52}$ D. R. Hamann, Phys. Rev. Lett., 76, 660 (1996).

${ }^{53}$ G.-M. Rignanese, A. De Vita, J.-C Charlier, X. Gonze and R. Car, Phys. Rev. B, 61, 13250 (2000).

${ }^{54}$ L. Levien, C. T. Prewitt and D. J. Weidner, Am. Mineral. 65, 920 (1980).

${ }^{55}$ M. C. Schabel, and J. L. Martins, Phys. Rev. B., 46, 7185 (1992).

${ }^{56}$ Y. Baskin, and L. Mayer, Phys. Rev., 100, 544 (1955).

${ }^{57}$ L. A. Girifalco, and R. A. Ladd, J. Chem. Phys. 25, 693 (1956).

${ }^{58}$ P. A. Serena, A. Baratoff, and J. M. Soler, Phys. Rev. B 48, 2046 (1993).

${ }^{59}$ G. Herzberg, Molecular Spectra and Molecular Structure. III. Electronic Spectra and Electronic Structure of Polyatomic Molecules, (Van Norstrand Reinhold, New York, 1966).

${ }^{60}$ P. Sautet (unpublished)
${ }^{61}$ G. Kresse anf J. Hafner, Phys. Rev. B 48, 13115 (1993). 